\title{
Role of Tumor Necrosis Factor-Alpha in Schizophrenia and Cognitive Impairment
}

\author{
Elmeida Effendy* (D), Mustafa Mahmud Amin (D), Nurul Utami \\ Department of Psychiatry, Faculty of Medicine, Universitas Sumatera Utara, Medan, Indonesia
}

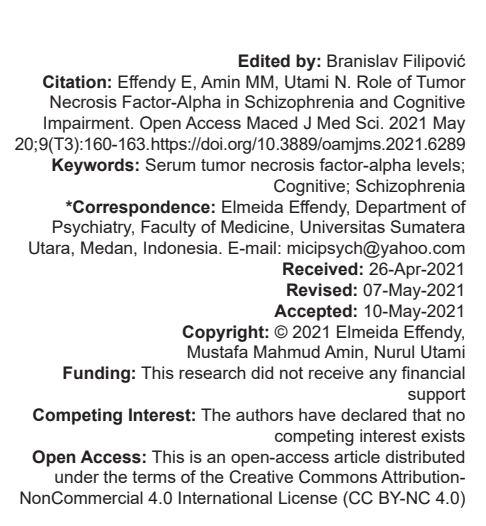

Abstract

BACKGROUND: Neuroinflammation and excitotoxicity play a key role as triggers and support for neurodegenerative processes, increase levels of tumor necrosis factor-alpha (TNF- $\alpha$ ) have been found in schizophrenic patients. Improved inflammatory processes have been demonstrated in acute and chronic schizophrenia. Schizophrenia is characterized by a collection of core symptoms that trigger individuals experiencing several cognitive disorders.

AIM: The objective of the study was to investigate the relationship of serum TNF- $\alpha$ levels to impaired cognitive function in schizophrenic patients in Medan, Indonesia.

METHODS: This research is cross-sectional analytical correlative study involving 40 male schizophrenic patients at Prof. Dr. M. Ildrem Mental Hospital through July to September 2019 that fulfilled our inclusion and exclusion criteria. Serum TNF- $\alpha$ levels were analyzed using Quantikine HS Human TNF- $\alpha$ Assay with a minimum detection limit of $0.1062 \mathrm{pg} /$ $\mathrm{ml}$ while, on the other hand, cognitive function test was carried out using the Mini-Mental State Examination (MMSE).

RESULTS: Serum TNF- $\alpha$ levels were found with a mean of 25.1216 with a standard deviation of 1.7629 . There is a positive correlation with moderate correlation $(r=0.4-<0.6)$ on cognitive function with the strength of correlation $r=0.434$.

CONCLUSION: We found that there is a link between serum TNF- $\alpha$ level and cognitive function as assessed with the MMSE.

\section{Introduction}

Psychotic disorders are a collection of a wide variety of conditions, ranging from positive, negative symptoms to cognitive problems such as concentration, processing speed, working, and long-term verbal memory and numerous aspects of executive functions which lead to impaired individual's functions [1]. Cognitive dysfunction actually does happen in nearly all schizophrenic patients (ODS) that affect numerous aspects of the patients life, including their capability of carrying out previously common or daily activities. In the extent of prognosis, cognitive performance serves as the key indicator, as well as, an important therapeutic concern [2].

It is very interesting that cognitive dysfunction is actually common, affecting nearly $75 \%$ of schizophrenic patients, but only $27 \%$ of these patients are considered of having normal neuropsychopathology [3]. Cognitive impairment occurs not only before the onset of psychotic symptoms and may remain even after remission [4]. The central characteristic in schizophrenia is known to be cognitive disorders and has been found to be linked with the severity of positive symptoms and is marginally correlated with the severity of negative symptoms. Furthermore, cognitive impairment is considered to be related to overall adherence to medication. Therefore, cognitive impairment is probably the nature of the schizophrenia itself which manifests in substantial decline in neurocognitive field, such as working and visual memory, speed of thinking, verbal and learning memory, attention and alertness, problem-solving, and reasoning [4].

Cognitive improvement can correct some difficulties to overcome negative symptoms, which supports the theory that there is a partial overlap in these symptoms [5].

Cognitive impairment in schizophrenia manifests in varying disorders, such as verbal fluency disorders (capability of generating spontaneous speech), learning sequence problem (from sequence of events), and decreased executive functioning (problems in maintaining concentration, prioritization, and modulation of behavior in accordance with specific social situation) [6].

In central nervous system, tumor necrosis factor-alpha (TNF- $\alpha$ ) plays a role in homeostasis and pathophysiology. In healthy people, TNF- $\alpha$ functions are an important physiological process such as synaptic plasticity, learning and memory, sleep, food and water intake, and strengthening astrocyte-induced synaptic [7]. 
The involvement of cytokines produced by microglia, astrocytes, neurons, and endothelial cells in the pathogenesis of different mental disorders, such as schizophrenia, acute depression, panic disorder, and autism, has been extensively studied. In a study conducted by Ajami et al., which examined the ratio of serum TNF- $\alpha$ and interleukin (IL)-10 concentrations in patients before and after treatment, there was a substantial decrease in serum TNF- $\alpha$ concentrations ( $p$ $=0.002$ ) and an increase in serum IL-10 concentrations $(p=0.008)$. Compared with healthy controls, the serum concentration of TNF- $\alpha$ in schizophrenic patients is higher. They concluded that increased TNF- $\alpha$ and decreased IL-10 might have an important role in the psychopathology of schizophrenia [8].

In a study conducted by Al-Asmari and Khan in Riyadh Saudi Arabia, TNF- $\alpha$ levels in schizophrenic patients $(27.04 \pm 4.11)$ were significantly higher than healthy controls $(17.25 \pm 3.30)$. They believe that by different pathways, the imbalance between proinflammatory and anti-inflammatory cytokines may be involved in the pathophysiology of schizophrenia. Cytokines are known to interfere with the neuroendocrine system, for example, the hypothalamicpituitary-adrenocortical system, the autonomic system (epinephrine and norepinephrine), and the neurotransmitter system (dopamine, serotonin, and glutamine). In addition, cytokines may either cross the blood-brain barrier (BBB) through the region of leakage or through active transport. Increased BBB permeability may allow immune or neurotoxic cytokines to invade the CNS and cause psychopathological alterations [9].

In patients with schizophrenia, elevated cytokine levels such as TNF- $\alpha$, transform beta growth factor, IL-6, IL-1 $\beta$ receptor, IL-1 blocker, IL-2 receptor, and IL-12 receptor have been identified; however, numerous studies have also found some controversial results. These variations relate to the determination of exclusion criteria, nature of the study, sample size, patient clinical status, metabolic and/or inflammatory disorder comorbidity, body mass index (BMI) potential effects, and smoking status may all cause differences in this variation [10].

Based on this background, the researchers wanted to find out whether there was a relationship between TNF- $\alpha$ and cognitive function in people with schizophrenia which was known based on the theory that there was still controversy in various studies.

\section{Methods}

\section{Participants}

The sample in this study was 40 people with schizophrenia included as subjects in the study.
All research subjects who had met the inclusion and exclusion criteria were asked for approval to take part in the study through the informed consent given. After obtaining approval, samples included in the inclusion criteria in this study were male schizophrenic patients who had been diagnosed based on the Guidelines for the Classification and Diagnosis of Mental Disorders in Indonesia III. Based on the first way of working, by determining the diagnosis using guidelines and structured interviews from Mini International Statistical Classification of Diseases-10. To determine cognitive function in the sample, a Mini-Mental State Examination (MMSE) test was performed.

In the inclusion criteria, schizophrenic male patients were taken from age between 20 and 40 years, and with BMI value of $18.50-24.99 \mathrm{~kg} / \mathrm{m}^{2}$, were willing to be respondents and could be interviewed. Exclusion criteria were set as follows, suffering from a mental disorder, having a family history of mental disorders, having a history of chronic medical illness, and a history of the use of alcohol and other substances (except caffeine and nicotine).

\section{MMSE assessment}

MMSE is a short test to assess cognitive function. MMSE was introduced by Folstein in 1975. MMSE is used to screen cognitive impairment patients, measure improvements in cognitive function overtime, and also to determine the influence of therapeutic agents on cognitive function. MMSE sensitivity and specificity are satisfactory with $83 \%$ sensitivity and $87 \%$ specificity. Administration of the test takes between 5 and $10 \mathrm{~min}$. MMSE assesses a number of cognitive domains, namely, time and place orientation, registration, attention and calculation, recall, and language consisting of naming objects, repetition of words, understanding and implementing verbal and written commands, writing, and copying images. Each assessment consists of several tests and is given a score for each correct answer. The total score on the MMSE if all the answers are correct is 30 . Based on interpretations, the score on the MMSE can be classified as: Normal: Score 25-30, mild: Score 20-24, moderate: Score 13-19, and weight: Score 0-12 [11].

\section{Statistical analysis}

This study is an observational empirical analysis with a cross-sectional approach. The ShapiroWilk test is used to assess normality of the data as the number of samples is $<50$. Furthermore, the data are analyzed to obtain the correlation value ( $r$ ). Data processing is done with the help of SPSS software. 


\section{Results}

Demographic descriptions of schizophrenic patients are presented in Table 1. Categorical variables are described in frequency and proportion, while numerical scale variables are presented in mean and standard deviation. It shows the characteristics of the study subjects based on the schizophrenic group and the control group. The mean age in the schizophrenic group was 33.80 with a standard deviation of 3.51 . Based on marital status most were with unmarried status, schizophrenic group as many as 27 $(21.60 \%)$. In education it was found in subjects with secondary education (57.50\%). The mean BMl in the schizophrenic group was 21.61 with a standard deviation of 2.01 .

Table 1: Demographic characteristics

\begin{tabular}{lcc}
\hline Variable & Mean \pm SD & $\mathrm{n}$ \\
\hline Age (years) & $33.8 \pm 3.51$ & \\
Marital status & & $13(10.40)$ \\
$\quad$ Married & & $27(21.60)$ \\
$\quad$ Unmarried & & \\
Education & & $23(57.50)$ \\
$\quad$ Middle education & & $17(42.50)$ \\
$\quad$ Higher education & $21.61 \pm 2.01$ & \\
Body mass index $\left(\mathrm{kg} / \mathrm{m}^{2}\right)$ & & \\
\hline
\end{tabular}

Table 2 shows the MMSE score with a mean of 25.1216 and a standard deviation of 1.7629. From the results of the normality test data using the Shapiro-Wilk $p=0.20(p>0.05)$, so it can be concluded that the data are normally distributed.

Table 2: Mean serum TNF- $\alpha$ levels in people with schizophrenia

\begin{tabular}{llll}
\hline Variable & Average & SD & p \\
\hline TNF- $\alpha$ & 25.1216 & 1.7629 & 0.20 \\
\hline
\end{tabular}

TNF-a: Tumor necrosis factor-alpha

Table 3 shows the MMSE score with a mean of 21.03 and a standard deviation of 5.21. From the results of the normality test data using the Shapiro-Wilk $p=0.07$ ( $p>0.05)$, so it can be concluded that the data are normally distributed.

Table 3: Average MMSE in people with schizophrenia

\begin{tabular}{llll}
\hline Variable & Average & SD & $p$ \\
\hline MMSE & 21.03 & 5.21 & 0.07 \\
\hline MMSE: Mini-Mental State Examination & &
\end{tabular}

Based on the results of the Pearson correlation test, serum TNF- $\alpha$ and MMSE scores obtained $p<0.05$ and concluded that there is a correlation between serum TNF- $\alpha$ levels and MMSE scores. The strength of the relationship between TNF- $\alpha$ and MMSE score of 0.434 shows a positive correlation with moderate correlation strength $(r=0.4-<0.6)$ (Table 4).

\section{Discussion}

This study is the first study conducted in Indonesia, especially in Medan, North Sumatra with the aim to find out the relationship between serum levels of TNF- $\alpha$ to the cognitive function of people with schizophrenia based on MMSE.
Table 4: Correlation of MMSE and serum TNF- $\alpha$ levels

\begin{tabular}{ll}
\hline Serum TNF- $\alpha$ levels & \\
\hline MMSE score & \\
& $p=0.434$ \\
$n=40$ & $n$ \\
\hline${ }^{*}$ Pearson, MMSE: Mini-Mental State Examination, TNF-a: Tumor necrosis factor-alpha
\end{tabular}

In Table 1, the mean age in the schizophrenic group was 33.80 with a standard deviation of 3.51 . This study is in accordance with research conducted by $\mathrm{Na}$ and $\mathrm{Kim}$ in Korea in 2007 to see monocytes, T-helper 1 and T-helper 2 in schizophrenic patients, they measured TNF- $\alpha$ levels in schizophrenic patients and healthy controls with each mean age and standard deviation are $32.86 \pm 12.76$ and $31.48 \pm 11.83$ [12].

Based on marital status, schizophrenic as many as $27(21.60 \%)$ subjects were not married. In people with schizophrenia, most $23(57.50 \%)$ have secondary education. Mean BMI $\left(\mathrm{kg} / \mathrm{m}^{2}\right)$ in the schizophrenic group was 21.61 with a standard deviation of 2.01. This is consistent with research conducted by $\mathrm{Na}$ and $\mathrm{Kim}$ in Korea in 2007 to see monocytes, T-helper 1 and T-helper 2 in schizophrenic patients found to have a mean BMI and the standard deviation in the schizophrenic group was $22.29 \pm 3.22$ and the group control $21.57 \pm 3.19$ [12]

Inconsistent evidence suggests enhanced cognitive function in schizophrenic patients with elevated TNF- $\alpha$ tumor. Due to discordant findings or the lack of trials, evidence for the role of other cytokines in cognitive dysfunction in schizophrenia patients is inconclusive [9]. There is a relationship between serum TNF- $\alpha$ levels and MMSE-dependent cognitive activity based on the findings of this analysis, which indicates a positive association with moderate correlation intensity $(r=0.4-<0.6)$.

We also acknowledge the limitation of this research is that, based on a study performed by Petrescu et al. in Romania, it does not regulate cigarette use patterns or history, showing that serum TNF- $\alpha$ levels were significantly higher in the smoker community compared to the non-smoker group. In addition, the serum concentration of TNF- $\alpha$ smokers who used more than 1 pack per day increased relative to those who got less than 1 pack per day. There is a strong association between TNF- $\alpha$ serum levels and exposure to tobacco smoke. They concluded that as a consequence of tobacco smoke exposure, high serum TNF- $\alpha$ levels in smokers showed an imbalance between proinflammatory and anti-inflammatory factors. Depending on the dosage of cigarettes, TNF- $\alpha$ concentrations rise in the serum of heavy smokers [13]. Studies previously found by Jiang et al. in 2013 and Zhang et al. in 2012 show that cognitive function is also impaired by smoking. In schizophrenic patients, smoking or nicotine can improve certain cognitive deficits, or nicotine works as a type of self-medication. This observation demonstrates that the etiology of cognitive deficits in schizophrenia may include dysfunction in the signaling of nicotine acetylcholine receptors [14], [15]. 


\section{Conclusion}

A total of 40 schizophrenic patients in Prof. M. Ildrem Mental Hospital were included in the study in July 2019-September 2019. We found that there is a relationship between serum TNF- $\alpha$ levels and cognitive function, based on the findings of this research based on MMSE which shows a positive correlation with moderate strength correlation $(r=0.4-<0.6)$.

\section{Authors' Contributions}

All authors contributed equally to this work.

\section{Acknowledgments}

The authors express their gratitude to all the staff of Prof. Ildrem Mental Hospital Medan in facilitating this research.

\section{References}

1. Urben S, Baumann P, Barcellona S, Hafil M, Preuss U, PeterFavre $\mathrm{C}$, et al. Cognitive efficacy of quetiapine in early-onset first-episode psychosis: A 12-week open label trial. Psychiatr Q. 2012;83(3):311-24. https://doi.org/10.1007/s11126-011-9201-3 PMid:22101738

2. Riedel M, Schmitz M, Østergaard PK, Ferrannini L, Franco MA Alfano $\mathrm{V}$, et al. Comparison of the effects of quetiapine extendedrelease and quetiapine immediate-release on cognitive performance, sedation and patient satisfaction in patients with schizophrenia: A randomised, double-blind, crossover study (eXtRa). Schizophr Res. 2015;162(1-3):162-8. https://doi. org/10.1016/j.schres.2014.12.027

PMid:25592805

3. Fisekovic S, Memic A, Pasalic A. Correlation between MoCA and MMSE for the assessment of cognition in schizophrenia. Acta Inform Med. 2012;20(3):186-9. https://doi.org/10.5455/ aim.2012.20.186-189 PMid:23322976

4. Hofer A, Fleischhacker WW. Pharmacological interventions. In: Roder V, Medalia A, editors. Neurocognition and Social
Cognition in Schizophrenia Patients Basic concepts and Treatment. Switzerland: Karger; 2010. p. 145-57. https://doi. org/10.1159/isbn.978-3-8055-9339-7

5. Riedel M, Spellmann I, Strassnig M, DouhetA, Dehning S, OpgenRhein $\mathrm{M}$, et al. Effects of risperidone and quetiapine on cognition in patients with schizophrenia and predominantly negative symptoms. Eur Arch Psychiatry Clin Neurosci. 2007;257(6):36070. https://doi.org/10.1007/s00406-007-0739-x

PMid:17629731

6. StahISM. Stahl's Essential Psychopharmacology Neuroscientific Basis and Practical Application. $4^{\text {th }}$ ed. New York: Cambridge University Press; 2013. p. 79-128.

7. Olmos G, Llado J. Tumor necrosis factor alpha: A link between neuroinflammation and excitotoxicity. Mediators Inflamm. 2014;2014:861231. https://doi.org/10.1155/2014/861231 PMid:24966471

8. Ajami A, Abedian F, Hosseini SH, Akbarian E, Navaei RA, Taghipour M. Serum TNF-alpha, IL-10 and IL-2 in schizophrenic patients before and after treatment with risperidone and clozapine. Iran J Immunol. 2014;11(3):200-9.

PMid:25265997

9. Al-Asmari A, Khan MW. Inflammation and schizophrenia: Alterations in cytokine levels and perturbation in antioxidative defense systems. Hum Exp Toxicol. 2014;33(2):115-22. https:// doi.org/10.1177/0960327113493305 PMid:23836841

10. Erguna S, Yanartas AO, Kandemira G, Yamanb A, Yildiz AM, Haklarb $\mathrm{G}$, et al. The relationship between psychopathology and cognitive functions with cytokines in clinically stable patients with schizophrenia. Psychiatry Clin Psychopharmacol. 2018;28(1):6672. https://doi.org/10.1080/24750573.2017.1380920

11. Kochhann R, Maria CO, Godinho C, Camozzato A, Chaves ML. Evaluation of mini-mental state examination scores according to different age and education strata, and sex, in a large Brazilian healthy sample. Dement Neuropsychol. 2009;3(2):88-93. https://doi.org/10.1590/s1980-57642009dn30200004 PMid:29213617

12. Na KS, Kim YK. Monocytic, Th1 and Th2 cytokine alterations in the pathophysiology of schizophrenia. Neuropsychobiology. 2007;56(2-3):55-63. https://doi.org/10.1159/000111535 PMid: 18037815

13. Petrescu F, Voican SC, Silosi I. Tumor necrosis factor-alpha serum levels in healthy smokers and nonsmokers. Int J Chron Obstruct Pulmon Dis. 2010;5:217-22. https://doi.org/10.2147/ copd.s8330

PMid:20714375

14. Jiang J, See YM, Subramaniam M, Lee J. Investigation of cigarette smoking among male schizophrenia patients. PLoS One. 2013;8(8):e71343. https://doi.org/10.1371/journal. pone.0071343 PMid:23977021

15. Zhang $X Y, C$ Chen DC, Xiu MH, Haile CN, Sun H, LuL, etal. Cigarette smoking and cognitive function in Chinese male schizophrenia: A case-control study. PLoS One. 2012;7(5):e36563. https://doi. org/10.1371/journal.pone.0036563

PMid:22570726 